\title{
Submitted: 15.10 .2019 Ultrasound assessment of extensor pollicis longus tendon Accepted: 10.11.2019 \\ Published: rupture following distal radius fracture: a sonographic and surgical correlation
}

\section{Keywords}

ultrasound, extensor pollicis longus, tendon, rupture, radius fractures

\author{
Louy Ghazal, Mohammed Nabi, Christopher Little, James Teh
}

\begin{abstract}
Department of Radiology, Nuffield Orthopaedic Centre, Oxford, UK
Correspondence: Department of Radiology, Nuffield Orthopaedic Centre Windmill Rd, Oxford OX3 7LD; tel.: 0300304 7777, e-mail: l.ghazal@hotmail.co.uk
\end{abstract}

DOI: $10.15557 / \mathrm{JoU} .2020 .0001$

\begin{abstract}
Aim of the study: To assess the features identified on ultrasound in patients presenting with suspected extensor pollicis longus tendon rupture, and correlate this with surgical findings. Material and methods: A retrospective case series review was performed in 11 patients ( 8 female, 3 male) with suspected extensor pollicis longus tendon rupture. All ultrasound examinations were performed by an experienced musculoskeletal radiologist using a highresolution linear-array probe. The study evaluated the associated sonographic appearances of extensor pollicis longus tendon rupture and correlated with surgical findings. Results: Rupture of the extensor pollicis longus tendon was identified in all cases proximally at the level of Lister's tubercle. The most common associated sonographic finding was an effusion in the sheath of compartment III (10 patients); 9 patients also had fluid within the sheath of compartment II. An empty tendon sheath was observed in 1 patient and tenosynovitis of the extensor pollicis longus sheath was demonstrated in 2 cases. In 9 patients, sonography revealed the retracted tendon ends as enlarged and hypoechoic with loss of their normal fibrillar appearance. Ultrasound depicted fracture involvement of Lister's tubercle in 8 cases (compared to 4 cases identified radiographically). In the 8 cases that proceeded to operative intervention, all of these were confirmed as extensor pollicis longus tendon rupture. Conclusion: Ultrasound is a valuable tool in identifying patients with extensor pollicis longus tendon rupture, providing the surgeon with invaluable details with regard to the level of rupture to aid pre-operative planning. US identifies associated ancillary features of extensor pollicis longus tendon rupture and reliably detects fractures involving Lister's tubercle.
\end{abstract}

\section{Introduction}

Extensor pollicis longus (EPL) tendon ruptures occur more commonly after undisplaced or minimally displaced distal radius fractures ${ }^{(1-3)}$. Rupture of the EPL tendon can develop without antecedent trauma, being reported in patients with rheumatic disease, following systemic or local steroid injection ${ }^{(4,5)}$ and in patients with carpometacarpal osteoarthritis ${ }^{(6)}$. Cortical irregularities and erosions result in roughening of the bone surface at the level of Lister's tubercle, which contributes to progressive attrition and consequent rupture of the EPL tendon ${ }^{(7)}$. Excessive and repetitive wrist motion in certain occupational groups can result in tendon friction against Lister's tubercle, predisposing it to rupture ${ }^{(8-10)}$. Iatrogenic tendon irritation can be caused by prominent screws after volar plate fixation ${ }^{(11)}$, against a dorsal plate or the pins of an external fixator ${ }^{(5)}$. Theories on the pathogenesis of EPL ruptures have been postulated in the literature to account for these clinical settings, which describe the interplay of anatomical, mechanical and vascular factors, making the tendon inherently vulnerable to rupture ${ }^{(1,3)}$.

Ultrasonography (US) plays an important role in the assessment for the presence of EPL tendon ruptures. US depicts the tendon in real time and allows rapid assessment of its integrity ${ }^{(12)}$, thereby providing a prompt 
diagnosis for the surgeon to plan their most appropriate course of action. The location of the incision is dependent on the level of the retracted ends, and this can be assessed on US to enable surgical planning. US can also influence the operative technique chosen by the surgeon, such that a direct primary repair of the tendon may not be possible if the tendon is found to be severely retracted or if the ends are atrophied or degenerated. A free tendon graft after harvesting palmaris longus if present, may then be used to augment a direct repair or bridge retracted ends. Alternatively, the tendon transfer technique which involves using extensor indicis to replace a non-salvageable EPL tendon usually confers a predictable outcome $\mathrm{e}^{(13,14)}$.

Ultrasound evaluation of the EPL tendon can be technically challenging for the sonographer due to the complex anatomy of the EPL within the extensor compartment and the importance of getting the diagnosis right for the patient and surgeon. In this paper, we describe the sonographic features of EPL rupture that can be identified, through a series of eleven cases which were correlated with surgical findings.

\section{Material and methods}

Written consent was obtained from the patients prior to submission for publication. A retrospective case series review was performed in 11 patients over a 2-year period at the John Radcliffe Hospital in Oxford, United Kingdom. Patients included were those referred from both orthopedic and plastic surgery specialties with suspected EPL tendon ruptures. For each patient, their age, gender and presumed cause of EPL rupture (trauma, non-traumatic and spontaneous causes) were recorded.

The ultrasound examinations were performed by a consultant musculoskeletal radiologist, with 20-year experience in musculoskeletal ultrasound. The procedure was performed using a $12 \mathrm{MHz}$ high-frequency linear-array transducer from a GE Logiq E9 ultrasound machine.

The examination commenced with both wrists palmar side down on the table, with the normal side acting as a control. The transducer was applied in the axial plane over the dorsal surface of the distal radius and the bony landmark of Lister's tubercle was identified. The EPL tendon within the $3^{\text {rd }}$ compartment was found on the ulnar side of Lister's tubercle. The tendon was followed proximally to the myotendinous junction and then distally along its full length as its curves round Lister's tubercle and crosses the tendons of the second compartment, terminating at its insertion onto the base of the distal phalanx of the thumb.

The findings identified on ultrasound were then correlated surgically using the operation note found in the patient's electronic record.

\section{Results}

Eleven patients were referred to radiology from the orthopedic and plastic surgery specialties. The average age of our patients was 51 years (age range 23-71 years). In this cohort of patients, 8 were female and 3 were male. The average interval between the US examination and the initial injury was 4.5 weeks. A total of 8 patients out of the 11 proceeded to operative intervention. The rest either refused surgical treatment or their function had improved following conservative management and did not require surgery (Tab. 1).

No visible tendon was seen in extensor compartment III at the level of Lister's tubercle in all 11 cases (Tab. 2). The average gap length was measured as $2.4 \mathrm{~cm}$ (gap range $1.4-3.6 \mathrm{~cm}$ ). The most common (10 patients) sonographic finding was effusion seen in the compartment III sheath (Fig. 1). There were 9 patients who had fluid also in the sheath of the extensor compartment II. An empty tendon sheath was observed in 1 patient (Fig. 2). Evidence of associated tenosynovitis of the EPL sheath was demonstrated in 2 cases with increased vascularity seen on Doppler interrogation (Fig. 3). In 9 patients, sonography revealed the retracted tendon ends as enlarged and hypoechoic with loss of their normal fibrillar appearance (Fig. 4). None of the cases demonstrated atrophic or degenerated changes of the tendon ends. In the 8 cases that proceeded to operative intervention, all of these were confirmed as EPL rupture. In 1 patient, the retraction gap was measured as $8 \mathrm{~cm}$ intra-operatively but was underestimated by US which measured the gap length as $3.6 \mathrm{~cm}$.

Of the 8 patients who sustained a fracture of the distal radius, radiography demonstrated the fracture line extending into Lister's tubercle in only half of these patients. In contrast, US depicted fracture involvement of Lister's tubercle (Fig. 5) in all of 8 cases and even identified a fracture of Lister's tubercle in 1 patient who had no visible fracture on the initial radiograph (Tab. 3).

Tab. 1. Summary of patient characteristics, time interval of US and surgical procedure

\begin{tabular}{|l|c|c|c|c|}
\hline $\begin{array}{l}\text { Patient } \\
\text { No. }\end{array}$ & Age & Gender & $\begin{array}{c}\text { Time to US after } \\
\text { injury (wks) }\end{array}$ & Operative technique \\
\hline 1 & 23 & F & 14 & EIP to EPL transfer \\
\hline 2 & 25 & M & 4 & EPL repair PL graft \\
\hline 3 & 54 & M & 2 & EIP to EPL transfer \\
\hline 4 & 47 & M & 4 & No operation \\
\hline 5 & 61 & F & 4 & No operation \\
\hline 6 & 35 & F & 2 & EIP to EPL transfer \\
\hline 7 & 71 & F & 3 & EIP to EPL transfer \\
\hline 8 & 70 & F & $<1$ & EIP to EPL transfer \\
\hline 9 & 63 & F & 4 & No operation \\
\hline 10 & 48 & F & 4 & EPL repair PL graft \\
\hline 11 & 65 & $f$ & 8 & EIP to EPL transfer \\
\hline $\begin{array}{l}\text { EIP - extensor indicis proprius; EPL - extensor pollicis longus; } \\
\text { PL - palmaris longus }\end{array}$
\end{tabular}


Tab. 2. Summary of US findings

\begin{tabular}{|l|c|}
\hline EPL Sonographic findings & Cases \\
\hline Level of rupture & 11 \\
\hline Proximal (Lister's tubercle) & 0 \\
\hline Distal (Distal phalanx) & $1.4-3.6$ \\
\hline Tendon end retraction & 2.4 \\
\hline Gap range (cm) & \\
\hline Gap average (cm) & 9 \\
\hline Tendon end state & 0 \\
\hline Enlarged and hypoechoic & 2 \\
\hline Atrophic ends \\
\hline Unremarkable appearances & 10 \\
\hline Tendon sheath & 9 \\
\hline Effusion $3^{\text {rd }}$ compartment & 1 \\
\hline Effusion 2 $2^{\text {nd }}$ compartment & 2 \\
\hline Empty tendon sheath & \\
\hline Tenosynovitis & 9 \\
\hline Lister's tubercle & 1 \\
\hline Fracture resulting in irregularity & 1 \\
\hline Variant anatomy & \\
\hline Smooth cortex & \\
\hline
\end{tabular}

In 1 patient, Lister's tubercle was seen as a variant in which both the radial and ulnar peaks were of similar heights (Fig. 6).

\section{Discussion}

Sonography identified complete rupture of the EPL tendon proximally at the level of Lister's tubercle in all patients, which was later supported by operative findings. De Maeseneer et al. (2008)(15) performed a sonographic correlation with anatomic features of the EPL tendon ruptures in 5 cadavers. They found a characteristic hypoechoic tubular area at the expected position of the EPL tendon, which corresponded with a dissection to the synovial sheath being distended by fluid, hemorrhage or scarring. In our study, this was not a consistent feature and is in keeping with Santiago et al. $(2008)^{(16)}$, who showed that tendon rupture tended to be illustrated by the absence of the tendon or tendon hypoattenuation.

Following confirmation of EPL rupture, sonography was able to identify the extent of proximal and distal tendon end retraction in all the cases. In evaluating the state of the retracted tendon ends, none of the cases revealed an atrophic appearance, which was not an expected finding as most cases were scanned less than 4 weeks following presentation. However, in many of our patients, the tendon ends appeared as thickened and hypoechoic stumps, which is a feature supported in previous literature ${ }^{(16)}$.

In our study, the ruptured EPL tendon sheath frequently contained a significant amount of fluid, and in several cases there was evidence of sheath thickening and neovascularity consistent with post-traumatic tenosynovitis. These

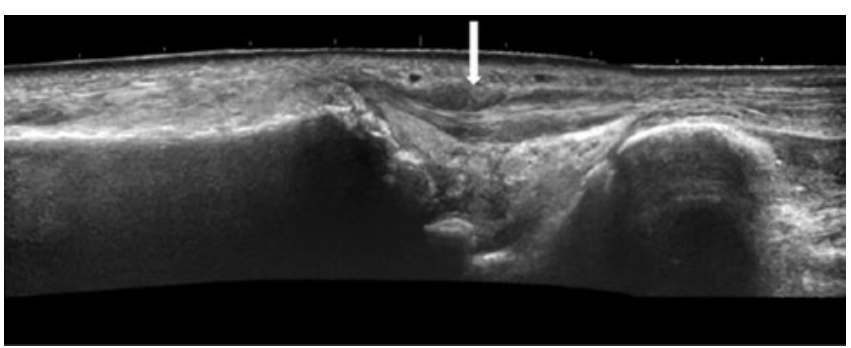

Fig. 1. Panoramic longitudinal sonogram at the level of the distal radius demonstrating effusion within compartment III sheath (arrow)

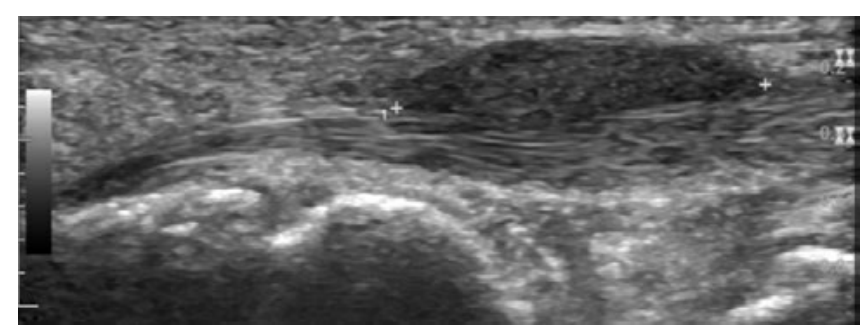

Fig. 2. Longitudinal image showing empty tendon sheath of the EPL depicted between cross markers, with the extensor carpi radialis longus seen deep to the EPL tendon sheath

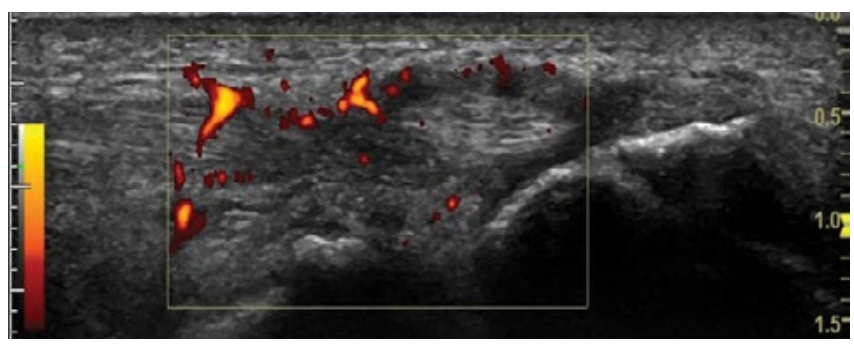

Fig. 3. Sonogram in the longitudinal plane illustrating neovascularity of the EPL tendon sheath on Doppler mode US

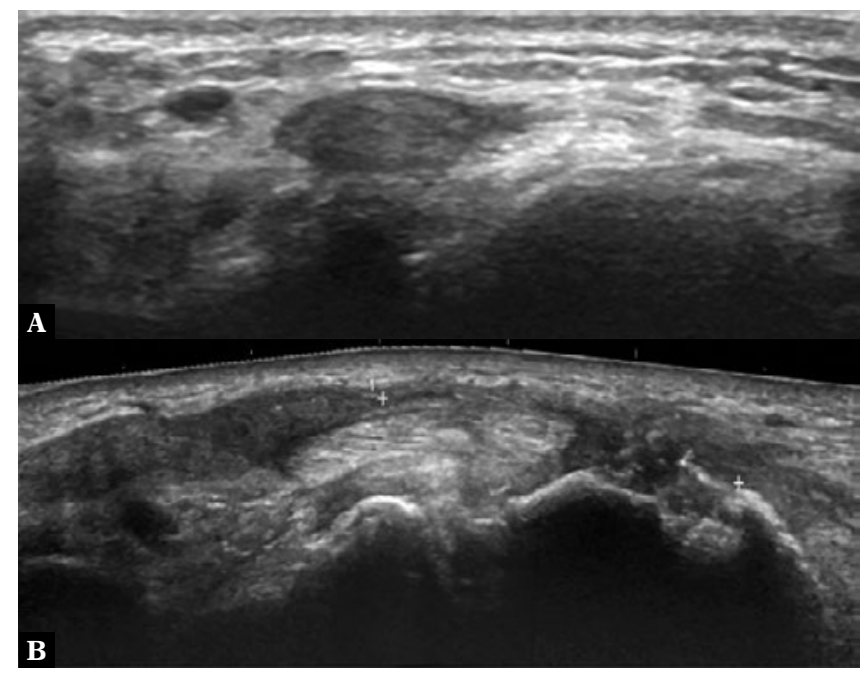

Fig. 4. Sonogram illustrating thickened hypoechoic appearance of the proximal retracted EPL stump in the transverse plane (A), and panoramic longitudinal view (B) illustrating that both the EPL tendon ends are retracted (cross markers), hypoechoic and enlarged 


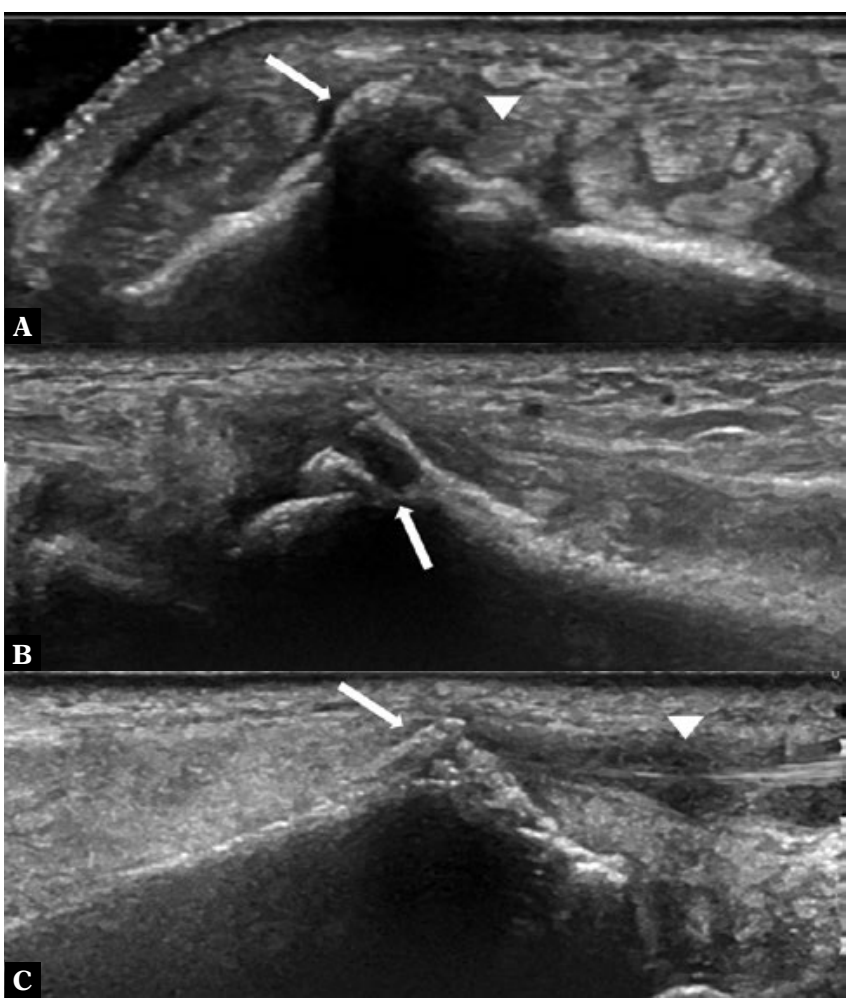

Fig. 5 A. Transverse ultrasound image illustrates a fracture extending through Lister's tubercle, resulting in an irregular dorsal cortex (arrow) and the EPL tendon in cross-section (arrow head) close to the fracture edge. B. Longitudinal sonogram demonstrates fracture of Lister's tubercle at the site of the EPL tendon rupture. C. Fracture extending through Lister's tubercle, resulting in dorsal cortex irregularity (arrow) and effusion within the EPL sheath (arrow head)

changes are likely accounted for by a secondary inflammatory response following rupture. The current study also frequently identified fluid within the $2^{\text {nd }}$ extensor compartment sheath, a finding supported by previous studies which have described a foraminal intercommunication between the sheaths of the EPL and extensor carpi radialis tendons $s^{(1,17,18)}$.

In most of the cases, EPL tendon rupture was associated with an undisplaced or minimally displaced distal radius fracture. Several previous studies ${ }^{(1-3)}$ have also found this association. It has been postulated that rupture ensues from increased pressure within the sheath that impedes blood flow in an already tenuous vascular part of the tendon adjacent to Lister's tubercle ${ }^{(1)}$. Helal et al. (1982) propose a similar theory, suggesting that an intact extensor retinaculum holding the EPL tendon closely against the fracture callus can lead to attritional rupture ${ }^{(2)}$.

In our study, sonography consistently identified an irregular cortical surface of Lister's tubercle. Fracture lines extending into Lister's tubercle were clearly demonstrated in all patients with distal radius fractures, and in one case US identified an occult fracture not depicted radiographically. The finding of an irregular Lister's tubercle supports the mechanical attrition theory for EPL rupture. Furthermore, this is an essential finding that should be communicated to the surgeon due to the implication of potential future risk to the tendon graft or tendon transfer, which may prompt the surgeon to smooth down the tubercle as a preventative measure.

Sonography incidentally identified an anatomical variant of Lister's tubercle in one patient, where both the ulnar and radial peaks were of similar height and size. Chan and Chong (2017) proposed a new system for anatomically classifying variants of Lister's tubercle based on size and morphology of its radial and ulnar peaks, which in our study would conform to the "Type 2 variant'. They suggest the 'Type 2 variant' predisposes the EPL to inherent microinstability and may result in increased risk of tendon rupture. Variant anatomy can also have surgical implications for pre-procedural planning of volar plate fixation, highlighting the importance of making a qualitative assessment of the morphology of Lister's tubercle ${ }^{(19)}$.

Several limitations were associated with the current study. As this was a retrospective study, the ultrasound examinations were performed only by a single operator. We found that US underestimated the extent of the retraction gap in one patient $(3.6 \mathrm{~cm})$ compared with surgical findings $(8 \mathrm{~cm})$; however, there was a delay of 8 weeks between the scan and the operation. Ideally, the retraction gap determined on US would have been correlated with the extent of retraction found operatively in all patients. However, surgeons do not routinely report the retraction gap in their operation notes. In addition, three patients did not proceed to operative repair, rendering it difficult to correlate with the sonographic determination of EPL rupture. Furthermore, the number of patients included in the study was small, which is a limitation also shared by other previous studies in the literature and reflects the fact that EPL rupture is not a common complication $(0.3 \%)^{(20)}$.

Tab. 3. Proportion of fractures involving Lister's tubercle identified on radiography and US

\begin{tabular}{|l|c|c|c|}
\hline Injury type & $\begin{array}{c}\text { No of cases identified } \\
\text { on radiography }\end{array}$ & $\begin{array}{c}\text { Fracture extends } \\
\text { into Lister's tubercle } \\
\text { on radiography }\end{array}$ & $\begin{array}{c}\text { Fracture extends } \\
\text { into Lister's tubercle } \\
\text { on US }\end{array}$ \\
\hline Undisplaced/minimally displaced distal radius fracture & 6 & 3 & 6 \\
\hline Displaced distal radius fracture & 2 & 1 & 2 \\
\hline Non-fracture injury & 3 & - & 1 \\
\hline
\end{tabular}




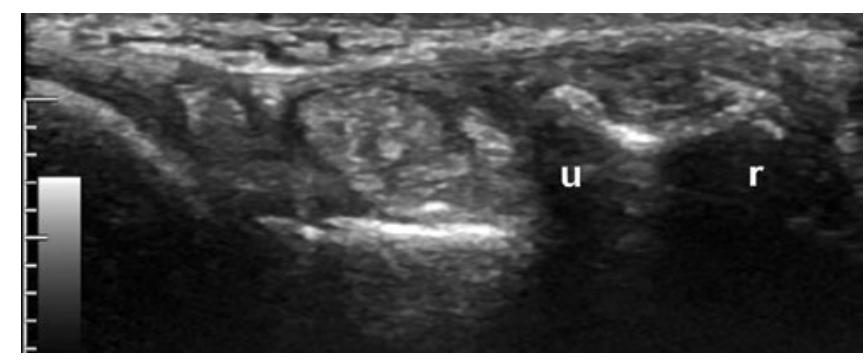

Fig. 6. Sonogram of the distal radius in the transverse plane illustrating the radial $(r)$ and ulnar $(u)$ peaks of Lister's tubercle of similar heights

\section{Conclusion}

Ultrasound is an excellent modality in detecting EPL tendon ruptures, enabling the operator to assess the extent of

\section{References}

1. Engkvist O, Lundborg G: Rupture of the extensor pollicis longus tendon after fracture of the lower end of the radius-a clinical and microangiographic study. Hand 1979; 11: 76-86.

2. Helal B, Chen SC, Iwegbu G: Rupture of the extensor pollicis longus tendon in undisplaced Colles' type of fracture. Hand 1982; 14: 41-47.

3. Hirasawa Y, Katsumi Y, Akiyoshi T, Tamai K, Tokioka T: Clinical and microangiographic studies on rupture of the E.P.L. tendon after distal radial fractures. J Hand Surg Br 1990; 15: 51-57.

4. Bonatz E, Kramer TD, Masear VR: Rupture of the extensor pollicis longus tendon. Am J Orthop (Belle Mead NJ) 1996; 25: 118-22.

5. Björkman A, Jörgsholm P: Rupture of the extensor pollicis longus tendon: a study of aetiological factors. Scand J Plast Reconstr Surg Hand Surg 2004; 38: 32-35.

6. Apard T, Marcucci L, Jarriges J: [Spontaneous rupture of extensor pollicis longus in isolated trapeziometacarpal arthritis]. Chir Main 2011; 30: 349-351. Doi: 10.1016/j.main.2011.08.004.

7. Hammert WC, Boyer MI, Bozentka DJ, Calfee RP: ASSH Manual of Hand Surgery. London. Lipincott Williams \& Wilkins, 2010.

8. Lloyd TW, Tyler MP, Roberts AH: Spontaneous rupture of extensor pollicis longus tendon in a kick boxer. British Journal of Sports Medicine 1998; 32: 178-179.

9. Taş S, Balta S, Benlier E: Spontaneous rupture of the extensor pollicis longus tendon due to unusual etiology. Balkan Med J 2014; 31: 105-106.

10. Choi JC, Kim WS, Na HY, Lee YS, Song WS, Kim DH et al.: Spontaneous rupture of the extensor pollicis longus tendon in a tailor. Clin Orthop Surg 2011; 3: 167-169. Doi: 10.4055/cios.2011.3.2.167.

11. Perry DC, Machin DMG, Casaletto JA, Brown DJ: Minimising the risk of extensor pollicis longus rupture following volar plate fixation of distal radius fractures: a cadaveric study. Ann R Coll Surg Engl 2011; 93: 57-60. Doi: 10.1308/003588411X12851639107151. tendon end retraction, state of the tendon ends, concomitant tendon changes of the EPL tendon and the neighboring extensor carpi radialis tendons. Fractures extending into Lister's tubercle resulting in a sharp, jagged cortex were frequently identified on ultrasound in our series of patients and have significant surgical implications.

\section{Conflict of interest}

We do not report any connection, either personal or financial with other individuals or organizations, which may be detrimental to the publication's contents or authorship rights.

12. Robinson P: Sonography of common tendon injuries. AJR Am J Roentgenol 2009; 193: 607-618. Doi: 10.2214/AJR.09.2808.

13. Wheeless C: Wheeless' Textbook of Orthopaedics. 2014: accessed 8.08.2018. Available from: http://www.wheelessonline.com.

14. Bullón A, Bravo E, Zarbahsh S, Barco R: Reconstruction after chronic extensor pollicis longus ruptures: a new technique. Clin Orthop Relat Res 2007; 462: 93-98. Doi: 10.1097/BLO.0b013e31806db4b6.

15. De Maeseneer M, Marcelis S, Jager T, Lenchik L, Pouders C, Van Roy P: Sonography of the finger flexor and extensor system at the hand and wrist level: findings in volunteers and anatomical correlation in cadavers. Eur Radiol 2008; 18: 600-607. Doi: 10.1007/s00330-007-0771-2.

16. Santiago FR, Plazas PG, Fernández JMT: Sonography findings in tears of the extensor pollicis longus tendon and correlation with CT, MRI and surgical findings. Eur J Radiol 2008; 66: 112-116. Doi: 10.1016/j. ejrad.2007.05.007.

17. Zbrodowski A, Gajisin S, Grodecki J: Intersynovial communication between the tendon sheaths of the extensor pollicis longus and extensor carpi radialis brevis muscles. J Hand Surg Br 1985; 10: 162-164. Doi: 10.1016/0266-7681(85)90006-3.

18. Cvitanic, OA, Henzie, GM, Adham M: Communicating foramen between the tendon sheaths of the extensor carpi radialis brevis and extensor pollicis longus muscles: imaging of cadavers and patients. AJR Am J Roentgenol 2007; 189: 1190-1197. Doi: 10.2214/ajr.07.2281.

19. Chan WY, Chong LR: Anatomical variants of Lister's tubercle: A new morphological classification based on magnetic resonance imaging. Korean J Radiol 2017; 18: 957-963. Doi: 10.3348/kjr.2017.18.6.957.

20. Hove LM: Delayed rupture of the thumb extensor tendon: a 5-year study of 18 consecutive cases. Acta Orthop Scand 1994; 65: 199-203. Doi: $10.3109 / 17453679408995434$ 